\title{
Atom Probe Tomography of Human Tooth Enamel and the Accurate Identification of Magnesium and Carbon in the Mass Spectrum
}

\author{
Alexandre La Fontaine ${ }^{1,2}$ and Julie Cairney ${ }^{1,2}$ \\ 1. School of Aerospace, Mechanical, Mechatronic Engineering, The University of Sydney, Australia. \\ 2. Australian Centre for Microscopy and Microanalysis, The University of Sydney, Australia.
}

According to the World Health Organization, $60-90 \%$ of children and nearly $100 \%$ of adults worldwide suffer from dental decay (caries), which occurs via the progressive dissolution of dental enamel. Human dental enamel is the hardest tissue in the body and plays a vital role in protecting teeth from wear and chemical attacks. It consists of a mineral phase, mainly in the form of highly oriented ribbon-like nanowires of carbonated hydroxyapatite [1].

It is well established that enamel mechanical strength and fatigue resistance is derived from its hierarchical structure, which consists of periodically-arranged bundles of HAP nanowires. However, we do not yet have a full understanding of the HAP crystallization process that leads to this structure. HAP crystallization is initiated by the mineralization of an amorphous calcium phosphate (ACP) precursor [2]. It was recently proposed that magnesium $(\mathrm{Mg})$ ions play a critical role in the stabilization of this ACP phase and the formation of the HAP mineral, where surface $\mathrm{Mg}$ ions retard the growth of HAP crystals, leading to the nanometer-sized HAP crystallites [3]. Knowledge of the distribution of Mg ions and the presence of the precursor ACP in mature human dental enamel would provide much needed information for a better understanding of enamel formation, and may eventually allow the development of strategies to enhance enamel repair and to slow or prevent caries.

The investigation of such structures in human dental enamel requires high chemical sensitivity at the atomic scale. Laser-assisted atom probe tomography (APT) provides position and identity of atoms with sub-nanometer resolution [4]. APT was recently used to reveal nano-structures in apatites [5, 6] and rodent tooth enamel [7, 8]. Inspired by this work, we examined human dental enamel using APT [9] and found Mg-rich ACP nanolayers between the HAP nanowires that make up the enamel. We also report Mg-rich elongated precipitates and pockets of organic material among the HAP nanowires. The correct chemical identification of the atoms and molecules field-evaporated from the precipitates and other nano-scale features was particularly challenging. This is mainly due to direct $\mathrm{Mg}$ and $\mathrm{C}$ peak overlap in the enamel mass spectrum. With the increasing interest in atomic scale characterization of biominerals using APT, there is a need for accurate identification of $\mathrm{Mg}$ and $\mathrm{C}$ in the APT mass spectrum.

Here we discuss the particular case of direct peak overlap between ${ }^{24} \mathrm{Mg}^{2+},{ }^{12} \mathrm{C}^{+}$and ${ }^{24} \mathrm{C}_{2}{ }^{2+}$ at 12 Dalton in human dental enamel. We show that the combined use of the isotopic distribution and spatial association with other ions enable to accurately differentiate $\mathrm{Mg}$ from $\mathrm{C}$ (Fig.1). We also highlight the contribution of $\mathrm{Mg}$, carbonates and organic carbon in the different nano-scale features detected in human dental enamel (Fig.2) [10].

\section{References:}

[1] A. Meckel, W. Griebstein, R. Neal, Archives of oral biology 10(5) (1965), 775.

[2] E. Beniash, R.A. Metzler, R.S. Lam, P. Gilbert, Journal of structural biology 166(2) (2009), 133. 
[3] H. Ding, et al, Crystal Growth \& Design 14(2) (2014), 763.

[4] B. Gault, et al, Atom probe microscopy, Springer Science \& Business Media (2012).

[5] L.M. Gordon, D. Joester, Nature 469(7329) (2011), 194.

[6] L.M. Gordon, L. Tran, D. Joester, ACS nano 6(12) (2012,) 10667.

[7] L.M. Gordon, et al, Science 347(6223) (2015), 746.

[8] L.M. Gordon, D. Joester, Frontiers in physiology 6, (2015).

[9] A. La Fontaine, et al, Science Advances 2(9) (2016), e1601145.

[10] The authors acknowledge the facilities and the scientific and technical assistance of the Australian Microscopy and Microanalysis facility (AMMRF) at the Australian Centre for Microscopy and Microanalysis (ACMM) at the University of Sydney.

a) C-rich region $\left(\mathrm{C}^{+}\right.$and $\mathrm{C}^{2+}$ detected $-\mathrm{Mg}^{2+}$ not detected $)$

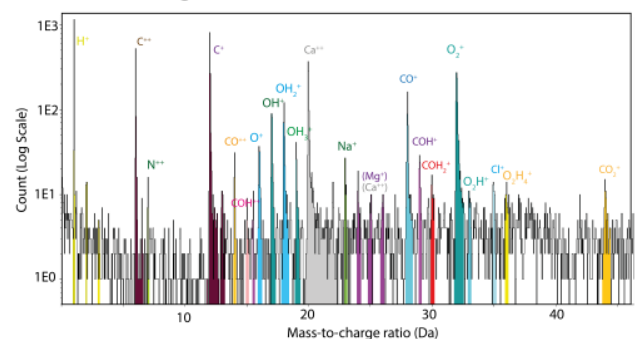

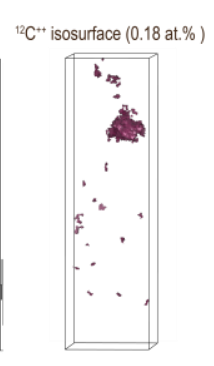

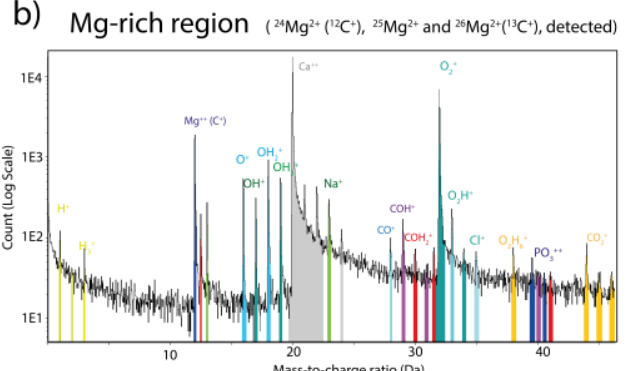

${ }^{24} \mathrm{Mg}^{+1}$ isosurface (1 at\%)

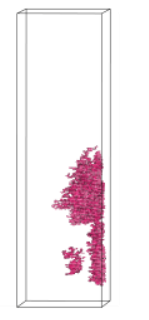

Figure 1. Atom probe mass spectra (0 Da to $48 \mathrm{Da}$ ) from (a) C-rich and (b) Mg-rich regions of human dental enamel.

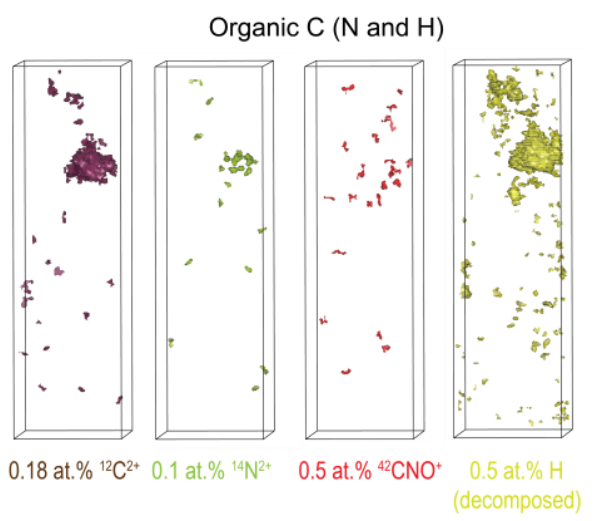

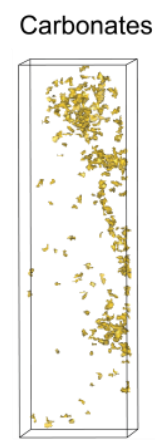

2 at. $\%{ }^{4} \mathrm{CO}^{+}$

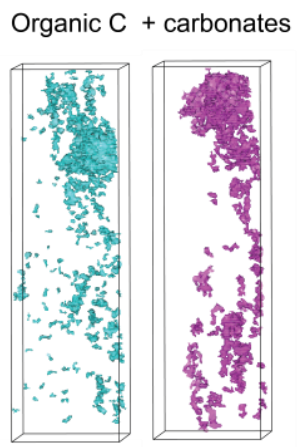

0.5 at. $\%{ }^{28} \mathrm{CO}^{+} 1.3$ at. $\%{ }^{29} \mathrm{COH}^{+}$

Figure 2. APT 3D reconstructed volumes from human tooth enamel with isosurfaces from the main species that arise from organic regions and carbonates. 